\title{
Portuguese Version of the DISABKIDS Diabetes Module ${ }^{1}$
}

\author{
Neuza da Silva \\ Institute for Health Services Research in Dermatology and Nursing (IVDP), University Medical Center Hamburg-Eppendorf \\ (UKE), Hamburg, Germany
}

Correspondence: n.dasilva@uke.de; Tel.: + 49 (0) 40741055 428; Fax.: + 49 (0) 40741040160

Received/Accepted: November 29, 2019

Key Words: DISABKIDS - Health-Related Quality of Life - Paediatric Diabetes.

The DISABKIDS questionnaires (1) were developed in 2006 simultaneously in seven European countries, namely Germany, the Netherlands, United Kingdom, France, Greece, Sweden and Austria. They include a set of developmentally appropriate and cross-culturally comparable questionnaires for assessing health-related quality of life (HrQoL) in children/adolescents between 8 and 18 years of age. The DISABKIDS tool set includes the Chronic Generic Module in its long and short forms (DCGM-37 and DCGM-12, respectively), as well as seven disease-specific modules for asthma, arthritis, cerebral palsy, cystic fibrosis, atopic dermatitis, diabetes, and epilepsy, all available in self- and proxy-report forms with established psychometric quality. Since then, the DISABKIDS questionnaires have been sequentially validated for additional European and non-European countries and they have been used in numerous clinical studies and health surveys.

\section{The DISABKIDS Diabetes Module (DDM) is Now Validated for Ten Countries}

A systematic search of the literature in three electronic databases (PubMed, PsycINFO, Web of Science; last search: 11/2018) with the keyword "disabkids" revealed 120 peer-reviewed original publications using the DISABKIDS questionnaires, 20 of which explicitly examined their measurement properties. Unsurprisingly, the DCGM-37 has been the most frequently used module, with sequential validation studies in Portugal, Brazil, Norway, Denmark and Japan. Among the condition-specific modules, the DISABKIDS Diabetes Module (DDM) was the most frequently used, with new psychometric studies in Sweden, Norway and Denmark, and now in Portugal (2).

As one of the most prevalent chronic health conditions in childhood, type 1 diabetes mellitus (T1DM) and its daily management pose significant psychosocial burden on children/adolescents and their families. The International Society for Pediatric and Adolescent Diabetes (ISPAD) recognizes quality of life as a central outcome in diabetes care

\footnotetext{
${ }^{1}$ Invited Commentary on "Assessing Paediatric HrQoL in Diabetes: Semantic and Pilot Validation Study of the Portuguese Versions of DISABKIDS-DM Condition-Specific Module” by Plaza et al.
} 
(3). Lower quality of life has been associated with poor glycaemic control, via less frequent blood glucose monitoring, with higher rates of depression and anxiety, and also with higher levels of family conflict related to the management of diabetes. Therefore, diabetes-specific questionnaires, which are more sensitive to detect clinically-significant differences/changes in $\mathrm{HrQoL}$ than generic measures, can be useful screeners to detect comorbid physical and/or psychological complications related to T1DM. By making available a new language version of the DDM, Plaza and colleagues provided a valuable contribution for further national and international clinical studies aimed at better understanding and improving the paediatric $\mathrm{HrQoL}$ and the quality of care for diabetes.

\section{DISABKIDS: More than an Instrument, a Well-Established Methodology}

The authors pointed out very clearly in their publication that the DISABKIDS is a well-established methodology for sequential cross-cultural validation of paediatric patient- and parent-reported outcomes (PRO). According to the COnsensus-based Standards for the selection of health Measurement INstruments (COSMIN), content validity is the most important measurement property of a PRO measure and the most challenging to assess (4). Defined as the degree to which the content of an instrument adequately reflects the construct to be measured, content validity includes the assessment of relevance (i.e., the relevance of each item for the construct being measured within a specific population/context), comprehensiveness (i.e., no key aspects of the construct are missing), and comprehensibility (i.e., the items are understood by patients and parents as intended).

The semantic validation of the Portuguese DDM, which included a general impression questionnaire and cognitive debriefings with patients and parents, ensured the relevance of the items for the targeted population, as well as the comprehensibility of items and response scales. The paediatric patients and parents also had the opportunity to suggest additional items, to guarantee that no culturally-specific key aspects of the construct were missing. In their qualitative analyses, the authors were sensitive to the cross-cultural equivalence of the original and the newly validated questionnaire and no major modifications of items and response scales were introduced. However, the qualitative results of this Portuguese study brought important insights for future international research aiming at improving the questionnaires.

\section{The Impact of the Paediatric Diabetes on the Family}

The lower HrQoL scores reported by the parents, compared to the child-reports, is well documented in paediatric literature (5). However, the reasons beyond this discrepancy are less studied. The cognitive debriefings performed by Plaza and colleagues suggest that the parents not only attributed a more intense emotional meaning to the items, but they also mirrored the impact on their own lives on their interpretations. For instance, the item 6 "Does it bother you that others can always eat and drink as much as they like?" was interpreted by one parent as his/her "major frustration, as a parent, not being able to give her [the child] all that she wants to eat or drink". These results raise the question about what are the parents really rating: their views about the child's HrQoL, impacted by their own concerns, or their own HrQoL impacted by the child's condition? Definitively, we are facing two different constructs that cannot be used indistinctly, but complementarily, as pointed out by the authors.

An additional contribution of this qualitative phase was the "need to be heard" unequivocally expressed by the Portuguese parents. Within a parent-child transactional approach, the impact of a paediatric chronic condition on the parents may be considered an important predictor of the children's HrQoL. In fact, other instruments have included the assessment of the impact on family, for example the PedsQL ${ }^{\mathrm{TM}}$ Family Impact Module or the Effects on Parents scale of the QoLISSY questionnaire (5). The parents' need to express their own feel- 
ings about the child's condition should be further explored for its universality or cultural specificity and perhaps integrated as part of the DISABKIDS questionnaires in a future conceptual revision.

\section{Conclusion}

The contribution of this study goes beyond the readiness of the 10th language version of the DDM and it paves the way for the field-test validation of the Portuguese questionnaires, for further national and international clinical studies, and for using the questionnaire in the Portuguese clinical setting as a screener to detect physical and psychological complications related to T1DM. Furthermore, it also instructs the improvement of the DISABKIDS instruments by including an additional module/scale assessing the impact of the condition on parents.

Conflict of Interest: The author declares that she has no conflict of interest.

\section{References}

1. The European DISABKIDS Group. The DISABKIDS questionnaires - quality of life questionnaires for children with chronic conditions. Lengerich: Pabst Science Publishers; 2006.

2. Plaza G, Barros L, Covinhas A, Carona C. Assessing paediatric Hrqol in diabetes: semantic and pilot validation study of the Portuguese versions of DISABKIDS-DM conditionspecific module. Central Eur J Paed. 2019;15(2):77-92.

3. Delamater AM, de Wit M, McDarby V, Malik JA, Hilliard ME, Northam E, et al. ISPAD Clinical Practice Consensus Guidelines 2018: psychological care of children and adolescents with type 1 diabetes. Pediatr Diabetes. 2018;19(Suppl 27):237-49.

4. Terwee CB, Prinsen CAC, Chiarotto A, Westerman MJ, Patrick DL, Alonso J, et al. COSMIN methodology for evaluating the content validity of patient-reported outcome measures: a Delphi study. Qual Life Res. 2018;27(5):1159-70

5. Quitmann J, Rohenkohl A, Sommer R, Bullinger M, Silva N. Explaining parent-child (dis)agreement in generic and short stature-specific health-related quality of life reports: do family and social relationships matter? Health Qual Life Outcomes. 2016;14(1):150. 